\title{
Vibration Controlling Techniques for Aircraft Hydraulic Pipe Excited by Pressure Pulsation Forces
}

\author{
Shunan Dai ${ }^{1, a}$, Yiqin Ling ${ }^{2, b}$, Yan $\mathrm{Su}^{2, *}$ \\ ${ }^{1}$ Shanghai Aircraft Airworthiness Certification Center, Shanghai, 200335, China \\ ${ }^{2}$ College of Civil Aviation, Nanjing University of Aeronautics and Astronautics, Nanjing, 210016, \\ China
}

aemail: daishunan_hd@caac.gov.cn, bemail: lingyiqin@nuaa.edu.cn, *email: suyannj@nuaa.edu.cn

Keywords: Hydraulic Pipeline; Pressure Pulsation; Tubular Joint; Dynamic Vibration Absorber

\begin{abstract}
In order to decrease the vibration of aircraft hydraulic pipeline, the vibration control techniques are studied from two aspects, i.e. suppression of the vibrating source and installation of dynamic vibration absorber. In this paper, the methods of increasing the length of the tubular joint and reduce the number of elbow are adopted to suppress the vibrating source, and a dynamic vibration absorber is developed to absorb the vibration of the main system. A finite element model of a real pipeline is established using ANSYS software and the exciting force is deduced under the pressure pulsation to study the response characteristics of the pipeline system. The results show that the designed pipe vibration can be reduced effectively after applying above methods.
\end{abstract}

\section{Introduction}

The hydraulic piping system [1][2] is an important part of the aircraft. Pipeline failure is becoming a prominent problem in military aircrafts. Excessive vibration might result in the oil leakage from the pipe joint, fatigue fracture, wearing of pipe wall, as well as the bursting of pipe wall. Oil leakage from the pipe joint has become the most frequent failure, which is caused by vibration for loose connection [3].

With the requirements of combat performance of modern military aircraft, the pressure of the hydraulic system has reached 21Mpa, and even 28Mpa.The pressure fluctuation caused by hydraulic piston pump has become the main excitation source of aircraft pipeline vibration [4]. According to the statistical results, the vibration fault caused by the pressure fluctuation accounted for more than $90 \%$ [5]. The greater the unevenness of pressure fluctuation, the larger the vibration force caused by pressure fluctuation. At the same time, the unreasonable pipe elbow and tube shape will cause the resonance of the pressure pulsation, which causes greater the pipeline vibration [6][7]. In order to decrease the vibration of aircraft hydraulic pipe excited by the pressure pulsation, this article studies the methods of suppressing vibration and maintain the natural frequency through modeling and simulation.

\section{Calculation and analysis methods of pressure pulsation in pipeline}

Before the analysis of the vibration of the hydraulic pipeline, it is necessary to carry out the corresponding analysis of the pressure pulsation. In this paper, an actual oil pipeline of a certain type of aircraft 1-166 is selected as the research object. Its pipe's (1Cr18Ni9Ti stainless steel) outer diameter $D$ is $18 \mathrm{~mm}$ and inner diameter $d$ is $16 \mathrm{~mm}$. The performance parameters of the pipe are as follows: the density $\rho=7850 \mathrm{~kg} / \mathrm{m}^{3}$, the modulus of elasticity $E=210 \mathrm{GPa}$, Poisson ratio $\mu=0.3$. Then we calculate the finite element by ANSYS. During the pretreatment, we carry out the grid division of pipelines by the way of automatic mesh division and the cell edge size is $0.01 \mathrm{~m}$. The finite element model is shown in Fig.1.

The working pressure of oil absorption pipeline is $21 \mathrm{Mpa}$. The pressure pulsation amplitude is $10 \%$ of the pressure in the pipeline, that is $\Delta P=2.1 \mathrm{Mpa}$. We can obtain the component of excitation force produced by the fluctuating pressure along the pipeline direction, 407.1N. The boundary 
conditions of the pipeline are shown in Fig.2. Elastic constraint are exerted at both ends of the pipe, whose support stiffness is $k=10^{7} \mathrm{~N} / \mathrm{m}$. Then two exciting forces are exerted along the direction of the pipeline at both ends of the elbow.

The excitation force of Pressure Pulsation is as bellow,

$$
R_{x 1}=S_{1} \Delta P=\frac{1}{4} \pi d_{1}^{2} \Delta P, \quad R_{x 2}=S_{2} \Delta P=\frac{1}{4} \pi d_{2}^{2} \Delta P
$$

In the type, $\Delta P$ is the fluctuating quantity of pressure in the pipeline, $\beta=\Delta P / P$ is the uneven pressure, $S_{1}$ and $S_{2}$ are the pipe flow area on both sides of the joint, $d_{1}$ and $d_{2}$ are the inner diameter of the pipeline on both sides of the joint [8].

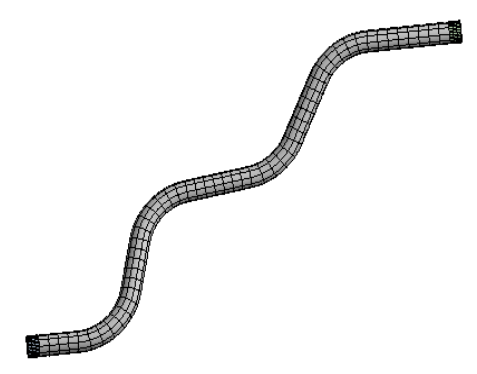

Fig.1. Mesh generation of Pipeline 1-166.

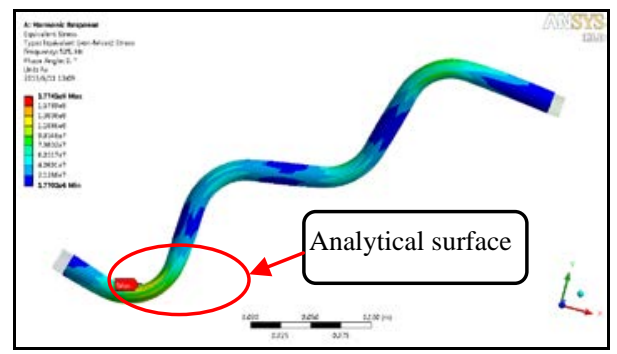

Fig. 3. Stress distribution of Pipeline 1-166 at Resonance.

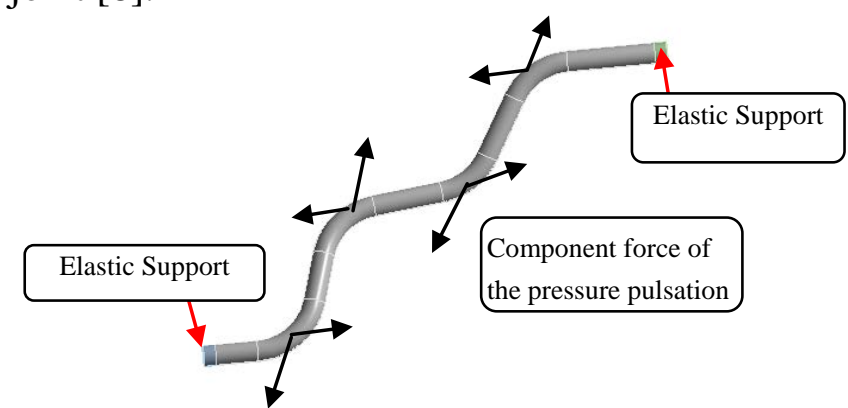

Fig. 2. Boundary conditions for harmonic response analysis of Pipeline 1-166.

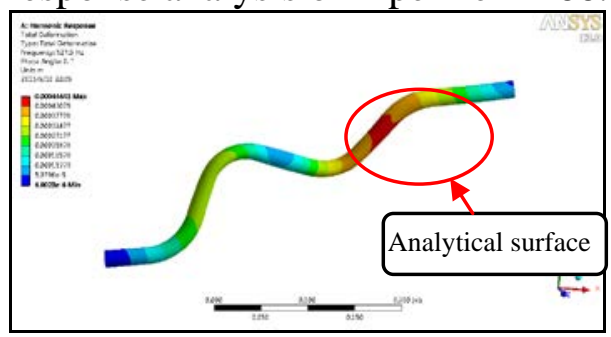

Fig. 4. Displacement distribution of Pipeline 1-166 at Resonance.

According to the real pressure fluctuation frequency range of the aircraft, the pipeline analysis frequency is $500-1000 \mathrm{~Hz}$. We set 500 solving points to carry out the harmonic response analysis. According to the results, the stress and displacement distribution of the entire model at the resonance point of the frequency range can be obtained, as shown in Fig.3 and Fig.4. In these two figures we can observe that the red area has occurred in two different regions which are the weakest stress and the maximum displacement.



(a) Comparison of the stress

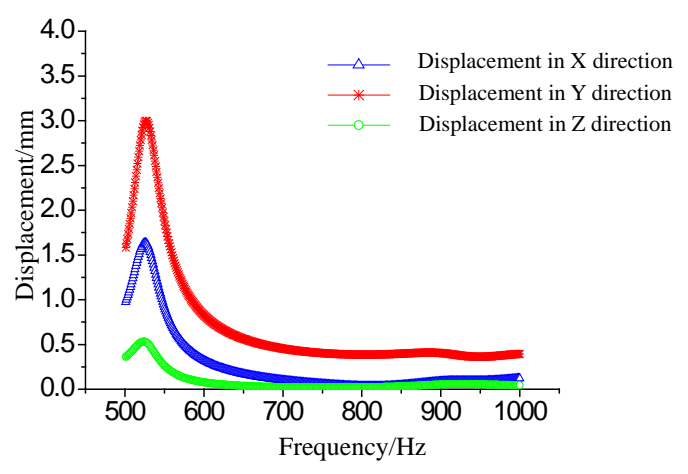

(b) Comparison of the stress

Fig. 5. Comparison of the pipeline 1-166 before and after changing the stress and displacement.

Then selecting the weakest stress point in Fig.3 as the analytical surface and calculating the stress response curve in three directions. And selecting the maximum displacement point as the analytical surface and calculate the displacement response curve in three directions. As shown in Fig.5. There is a resonating frequency which is $526 \mathrm{~Hz}$ between $500 \mathrm{~Hz}$ and $1000 \mathrm{~Hz}$. At this 
instance, the maximum stress is present in $\mathrm{Z}$ and $\mathrm{Y}$ direction in the corresponding analysis.

\section{Analysis of the influence on pressure pulsation caused by the change of pipe}

Changing the specific shape of the pipeline is the main mean to suppress the vibration of the pipeline. In this paper, the methods of increasing the length of the joint and reducing the number of elbow are used to change the shape of pipeline.

\section{Increase the length of the joint}

In the view of the problem of large vibration in the oil suction pipe system in the actual aircraft piping system, this section of the pipeline is selected as the research object. Then we change the sixth joint coordinates of the oil absorption tube 1-166 and keep the other unchanged. As shown in TABLE I, we can form the pipeline 2-166, that is to increase the length of the joints of the pipe and to compare the stress and displacement caused by the pressure pulsation before changing the pipe and after changing the pipe shape respectively [9] .

\section{TABLE I}

Joint coordinates of pipeline

\begin{tabular}{c|cccccc}
\hline $\begin{array}{c}\text { Joint No. } \\
\text { Type of Pipe }\end{array}$ & 1 & 2 & 3 & 4 & 5 & 6 \\
\hline $1-166$ & $(0,0,0)$ & $(85.10,0,0)$ & $(119.02,113.33,0)$ & $(239.27,126.82,0.28)$ & $(295.56,219.69,31.57)$ & $(409.40,217.25,41.54)$ \\
\hline $2-166$ & $(0,0,0)$ & $(85.10,0,0)$ & $(119.02,113.33,0)$ & $(239.27,126.82,0.28)$ & $(295.56,219.69,31.57)$ & $(557.74,202.93,53.38)$ \\
\hline
\end{tabular}

Selecting the pipeline 1-166 before modification and the pipeline 2-166 after modification to study. As shown in Fig.6, the outer diameters D of the both pipelines is $18 \mathrm{~mm}$, the inner diameters $\mathrm{d}$ is $16 \mathrm{~mm}$ and they are all $1 \mathrm{Cr} 18 \mathrm{Ni9Ti}$ stainless steel pipes. After grid division, we exert elastic constraint at both ends of the pipe whose the supporting stiffness $\mathrm{k}$ is $10^{7} \mathrm{~N} / \mathrm{m}$. Besides we also exert two exciting forces which are along the direction of pipe line at both ends of the elbow, 407.1N.

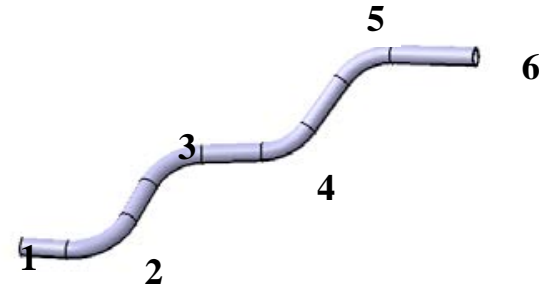

(a) Pipeline 1-166

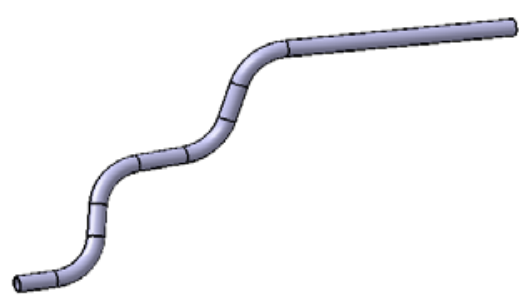

(b) Pipeline 2-166

Fig. 6. Modified oil pipeline of No.1.

Setting up the analysis frequency of the pipeline as $500-1000 \mathrm{~Hz}$ and define a total of 100 points. Then calculating the harmonic response of the pipeline under the pulse excitation so that we can get the stress distribution and displacement of the model at the resonance point of the frequency range. We select the weakest stress and the maximum displacement areas of these two pipelines as analytical plane. The results obtained by the analysis are shown in Fig.7.

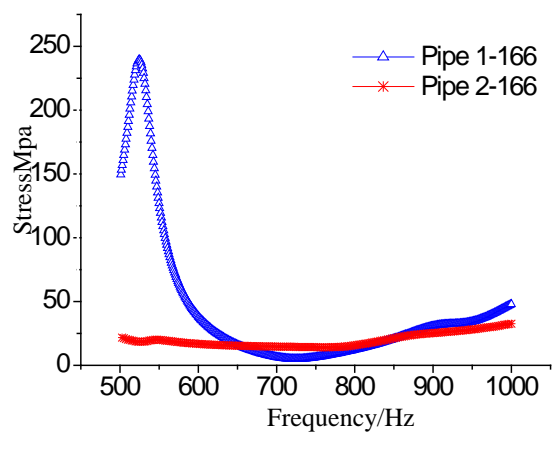

(a) Comparison of the stress

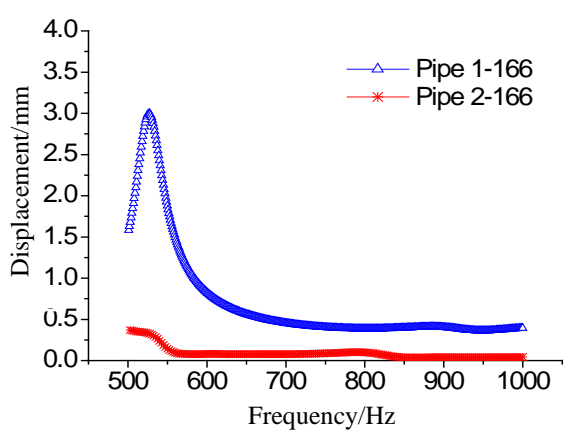

(b) Comparison of the displacement

Fig. 7. Comparison of the pipeline 166 before and after changing the stress and displacement.

We can see from Fig.7 (a) that the stress in the pipeline 2-166 is significantly smaller than that of the 1-166 when exerted elastic constraint at both ends. From Fig.7 (b), it can be seen that the displacement of the pipeline 2-166 after the change is smaller than that of the pipeline 1-166 and the 
stress and displacement are obviously decreased after the change. This shows that there should be a sufficient length at the joint of the pipe in the design.

\section{Reduce the number of elbow}

Because the pressure pulsation is mainly generated in the elbow, so we try to reduce the number of elbow. We removed the fourth joints of pipeline 1-166 and keep the other unchanged. Then the pipeline 3-166 pipeline is formed, as shown in Fig.8. When we analyze the harmonic response of two pipes, we exert elastic constraint at both ends of the pipe and the supporting stiffness $k$ is $10^{7} \mathrm{~N} / \mathrm{m}$ after grid division. We also exert two exciting forces, measuring $407.1 \mathrm{~N}$ each, which are along the direction of pipeline at both ends of the elbow. Next, the harmonic response is calculated, so that we can know the reduction of the number of elbow can effectively reduce the pressure pulsation, and suppress the vibration of the pipeline.



(a) Pipeline 1-166



(b) Pipeline 3-166

Fig. 8. Modified oil pipeline of No.2.

Setting up the analysis frequency of the pipeline as $500-1000 \mathrm{~Hz}$ and define a total of 200 points. Then calculating the harmonic response of the pipeline under the pulse excitation so that we can get the stress distribution and displacement of the model at the resonance point of the frequency range. We select the weakest stress and the maximum displacement areas of these two pipelines as analytical plane. The results obtained by the analysis are shown in Fig.9.

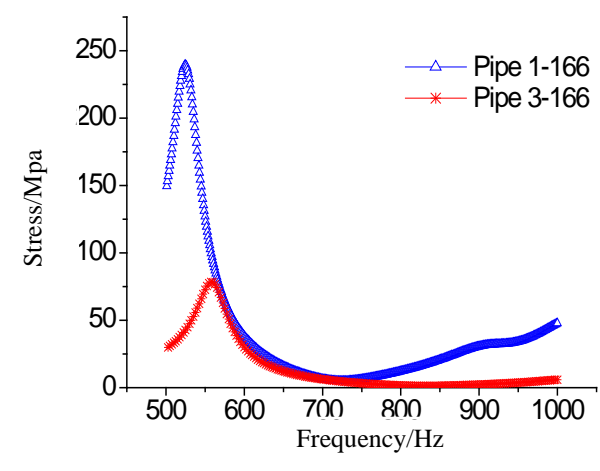

(a) Comparison of the stress



(b) Comparison of the displacement

Fig. 9. Comparison of the stress and displacement of Pipeline 166 before and after reduced elbow.

As can be seen from Fig.9, the vibration of pipeline stress is significantly reduced after reducing the number of bent pipe. However, the displacement change is not very big. This shows that, for oil absorption pipeline, reducing the number of elbow to change the tube can effectively reduce the vibration of the pipeline, but not necessarily the displacement amplitude.

\section{Analysis of the influence of dynamic vibration absorber on the pressure fluctuation of the pipeline}

The natural frequency of the pipe in the pipe system has been determined. If the tube subjected to the excitation frequency is close or relatively close with the natural frequency of the tube, it will lead to mechanical resonance of the pipe. At this time, the vibration can be reduced by adding a power absorbing device, which provides the same but the contrary control force as the main vibration force of the main system [7][8]. In this paper, the vibration characteristics of the oil absorption tube 1-166 are designed. Because the pipe is cylindrical, the characteristics of circumferential direction are presented, so the design of the vibration absorber with the characteristics of the vibration characteristics is needed. The vibration absorber structure is 
designed as show in Fig.10. It is composed of two external quality rings, two internal quality rings, four dampers and springs in the middle. The inner ring of the absorber is held on the pipe through the bolt clamp, and the results indicate the good performance of such a structure for vibration reduction.

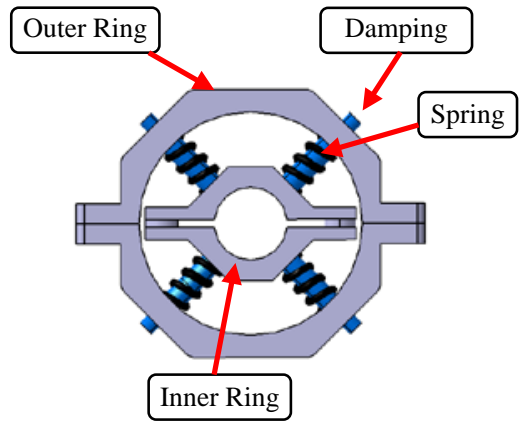

Fig. 10. Model of dynamic vibration absorber.

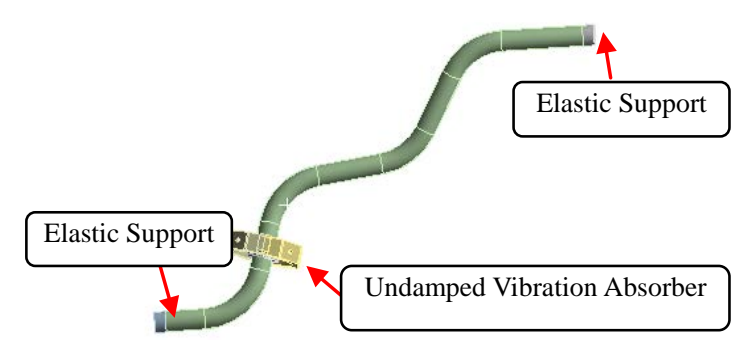

Fig. 11. Location of installed dynamic vibration absorber.

The designed vibration absorber is clamped in a large part of the pipe vibration, and the elastic constraint is applied at both ends of the pipe that the supporting stiffness is $k=10^{7} \mathrm{~N} / \mathrm{m}$. The vibration force of the pipe at both ends of the elbow is applied to the pipe elbow at the end of the pipe elbow, and the vibration force is $407.1 \mathrm{~N}$, as shown in Fig. 11.Due to the ratio of natural frequency between dynamic vibration absorber's damper and main structure is 1.0 , and the damping according to the actual requirements, it is necessary to adjust the stiffness and damping of the spring to achieve a good damping effect .

The quality of the pipe $m_{1}=212 \mathrm{~g}$, in order to reduce the resonance peak of $562 \mathrm{~Hz}$ in Fig.6 (a), the parameters of the absorber spring, mass $m_{2}=18 \mathrm{~g}$,spring stiffness $k_{2}=7.27 \mathrm{e} 4, \omega_{2}=524.6 \mathrm{~Hz}, \zeta_{2}=0.030$. The analysis frequency of the pipeline is designed of $500-1000 \mathrm{~Hz}$, and a total of 200 solving points are defined, and the harmonic response of the pipeline 1-166 in the vibration excitation is obtained respectively.

From Fig. 12, it can be seen that the vibration stress and the displacement of the pipeline are obviously reduced after installing the dynamic vibration absorber, which shows that the dynamic vibration absorber has a good control effect on the main structure.

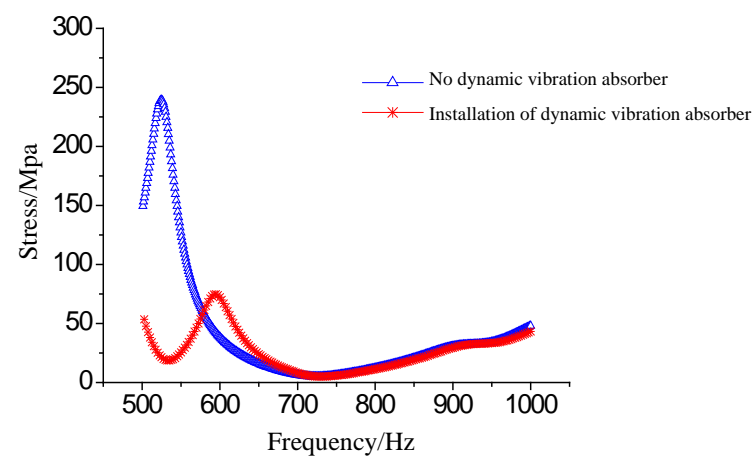

(a) Comparison of the stress

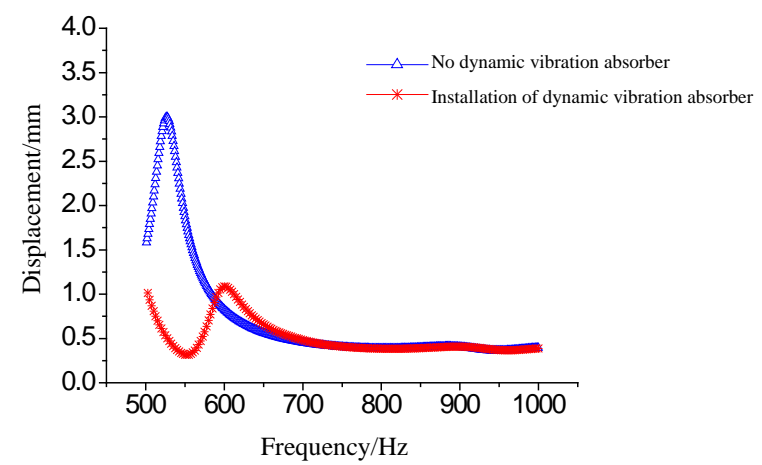

(b) Comparison of the displacement

Fig. 12. Comparison of the pipeline 1-166 before and after installing dynamic vibration absorber.

\section{Conclusion}

Taking the actual aircraft hydraulic pipeline as the research object, and according to the actual problem of the pipeline vibration, two methods of restraining the vibration of the pipeline are summarized, and the finite simulation analysis is carried out on these two methods. Upon the two methods, the pipeline pressure pulsation can be reduced effectively by changing the tube shape, such as changing the length of the joint and reducing the number of elbow, and the inhibitory effect of pipeline vibration source can be achieved. It can be provided the theoretical guidance and basis 
for restraining the vibration of the pipeline. Moreover, the dynamic vibration absorber has advantages of simple structure, convenient frequency modulation and installation. Using the principle of dynamic vibration absorber, the vibration of the pipe is installed in a large position, which can reduce the vibration level of the pipe.

\section{Acknowledgement}

This work was financially supported by the National Natural Science Foundation (U1233114). It was also supported in part by AVIC Commercial Aircraft Engine Co., Ltd.

\section{References}

[1] Han Zhi-yong, He Ren-mu, Xu Yan-hui. Study on Resonance Mechanism of Power System Low Frequency[J]. Proceedings of the CSEE,2008,28(1):47-51.

[2] Oscillation Induced by Turbo-pressure Pulsation[J]. Proceedings of the CSEE, 2008, 28(1): 47-51.

[3] Dang Xi-qi, Chen Shou-wu. The Air-current Pulsation and Pipeline Vibration of Piston Compressor[M].XI’AN Jiaotong University Press, 1984.57-65.

[4] Wang Qiu-ying. Pressure Pulsating Analysis of Pipeline Equipment[J]. Manufacturing Technology, 2007, (8): 48-50.

[5] Frederick Scheideman, Michael Schary, Rajendra Singh. Thermo dynamic and Acoustic Simulation of Positive Displacement Refrigeration Compressors[C]. Purdue Compressor Technology Conference, 1978:290-299.

[6] The research team of pipeline vibration in Xi'an Jiaotong University. Air Flow Pulsation and Pipe Vibration in the Pipeline of Reciprocating Compressor[J]. Journal of Xi'an Jiaotong University, 1978, (1):61-86.

[7] Zhou Yun, Liu Ji. Vibration and Damping of Piping Systems[J]. Journal of Harbin University of Civil Engineering and Architecture, 1994, 27(5):108-114.

[8] Hu Hai-yan, Sun Jiu-hou, Chen Huai-hai. Mechanical Vibration and Shock[M]. Beijing: Aviation Industry Publishing House, 2002. 82-87.

[9] Zeng Sheng, Ren Yi, Cheng Tao-tao, Zheng Shuiying. Vibration Suppression of Pipe System with Tuned Mass Damper[J]. Journal of Vibration, Measurement \& Diagnosis, 2012, 32(5): 823-826. 